\title{
Risk factors of brain metastasis during the course of EGFR- TKIs therapy for patients with EGFR-mutated advanced lung adenocarcinoma
}

\author{
Xiaoyan Ma1,2,3,*, Hui Zhu' ${ }^{2, *}$, Hongbo Guo ${ }^{4}$ Anqin Han', Haiyong Wang ${ }^{2}$, Wang \\ Jing $^{2}$, Yan Zhang ${ }^{5}$, Li Kong ${ }^{2}$ and Jinming $\mathbf{Y u}^{2,3}$ \\ ${ }^{1}$ School of Medicine and Life Sciences, University of Jinan, Jinan, Shandong, China \\ 2 Department of Radiation Oncology, Shandong Cancer Hospital Affiliated to Shandong University, Jinan, Shandong, China \\ ${ }^{3}$ Shandong Academy of Medical Sciences, Jinan, Shandong, China \\ ${ }^{4}$ Department of Thoracic Surgery, Shandong Cancer Hospital Affiliated to Shandong University, Jinan, Shandong, China \\ ${ }^{5}$ Department of Medical Oncology, Shandong Cancer Hospital Affiliated to Shandong University, Jinan, Shandong, China \\ * These authors have contributed equally to this study \\ Correspondence to: Jinming YU, email: sdyujinming@126.com
}

Li Kong, email: Kongli7@sina.com

Keywords: brain metastasis, prophylactic cranial irradiation, risk factors, epidermal growth factor receptor, advanced lung adenocarcinoma

Received: April 11,2016 Accepted: September 02, $2016 \quad$ Published: September 08, 2016

\section{ABSTRACT}

Controversial value of prophylactic cranial irradiation (PCI) in NSCLC in terms of survival benefit prompted us to explore the possible risk factors for brain metastasis (BM) during the course of EGFR-TKIs therapy from EGFR-mutated advanced lung adenocarcinoma and identify the potential population most likely to benefit from PCI, because BM remains a therapeutically challenging issue. We retrospectively reviewed the records of 134 patients with EGFR-mutated advanced lung adenocarcinoma between 2008 and 2012. The cumulative incidence of BM was calculated by the Kaplan-Meier method, and Multivariate Cox regression analysis was used to assess the independent risk factors for BM. Thirty-four patients (34/134, 25.4\%) developed BM during the course of EGFR-TKIs therapy. Moreover, the Multivariate analysis indicated that age $\leq 53$ years (HR: 2.751, $95 \%$ CI: 1.326-5.707; $p=0.007$ ), serum carcinoembryonic antigen (CEA) $\geq 23 \mathrm{ng} / \mathrm{mL}$ (HR: 3.197, $95 \%$ CI: 1.512-6.758; $p$ $=0.002$ ) and EGFR exon 21 point mutations (HR: 2.769, $95 \%$ CI: 1.355-5.659; $p=$ 0.005 ) were the independent high-risk factors for developing BM, which could offer important insights into the individualized treatment. Further studies are warranted to validate our findings.

\section{INTRODUCTION}

Lung cancer, including non-small cell lung cancer (NSCLC) and small cell lung cancer (SCLC), is the leading cause of cancer death all over the world [1]. Advanced lung adenocarcinoma is the predominant type of NSCLC and BM is a common problem in NSCLC with a dismal prognosis. Approximately $10 \%$ of NSCLC patients present with BM at their initial diagnosis and 40$50 \%$ of NSCLC patients develop BM during the course of the disease [2]. BM, undoubtedly, exerts a devastating influence on survival and quality of life of these patients; the median overall survival (mOS) was reported to be merely 4-6 months after whole brain radiation therapy (WBRT) [3, 4]. Therefore, special attention should be paid to reduce the incidence of $\mathrm{BM}$.

For patients with EGFR-mutated NSCLC, a large amount of clinical trials have demonstrated the overwhelming superiority of EGFR-TKIs as a first-line treatment over the routine chemotherapy according to response rate (RR) and progression-free survival (PFS) [5-10]. In addition, Sperduto et al. have revealed that EGFR mutations profoundly influenced the survival of patients with BM from lung adenocarcinoma, presenting 
EGFR-positive patients with the median survival time of 25.1months [11]. It has also been reported that EGFRTKIs are effective in BM from EGFR-mutated NSCLC [12-14]. Indeed, EGFR-TKIs could pass through brainblood barrier (BBB) and accumulate in brain metastatic lesions [15]. However, there remain some patients developing BM during the course of EGFR-TKIs therapy. Lee et al. [16] found that $26 \%$ developed central nervous system (CNS) failure and 13\% experienced isolated CNS failure among 166 patients with a clinical benefit to EGFR-TKIs.

Recently, the survival benefit conferred by PCI in SCLC has been established. With regard to NSCLC, PCI can decrease the occurrence of BM, whereas uncertainties still remain regarding to the survival advantage [17]. To our knowledge, RTOG-0214 is a randomized controlled trial to explore the value of PCI in NSCLC on the basis of combined-modality therapy [17]. However, the study was closed early due to slow accrual and reached only a third of the targeted accrual. Moreover, it failed to improve OS and its outcome should be interpreted cautiously, which suggests that PCI should be targeted patients with the highest risk for BM and based on satisfactory locoregional and extracranial control. Therefore, we suppose that PCI could bring survival benefit for patients with a high risk of BM during the course of EGFR-TKIs therapy from EGFRmutated advanced lung adenocarcinoma.

To date, previous results on risk factors of BM in NSCLC are not completely consistent, which included non-squamous cell carcinoma [18], younger age [18-20], high serum CEA level [21], no adjuvant chemotherapy [19,
22] and various disease stages [19, 22]. Notably, patients with EGFR-mutated pulmonary adenocarcinoma show a higher likelihood of BM than those with wild-type EGFR [23]. However, the risk factors for developing BM during the course of EGFR-TKIs therapy from EGFR-mutated advanced lung adenocarcinoma were rarely evaluated.

In the present study, we reviewed 134 patients with EGFR-mutated advanced lung adenocarcinoma, investigated the possible risk factors for developing BM during the course of EGFR-TKIs therapy and tried to identify the potential patients most likely to benefit from PCI.

\section{RESULTS}

\section{Patient characteristics}

For 134 patients included in this study, patient characteristics are shown in Table 1. The median age was 59 years (range: 35-81 years). Among 134 patients with EGFR-mutated advanced lung adenocarcinoma, $34(25.4 \%)$ patients developed BM during the course of EGFR-TKIs treatment and $100(74.6 \%)$ patients didn't.

\section{Survival}

Follow-up was completed in all 134 patients until December 2015, and the median follow-up period was

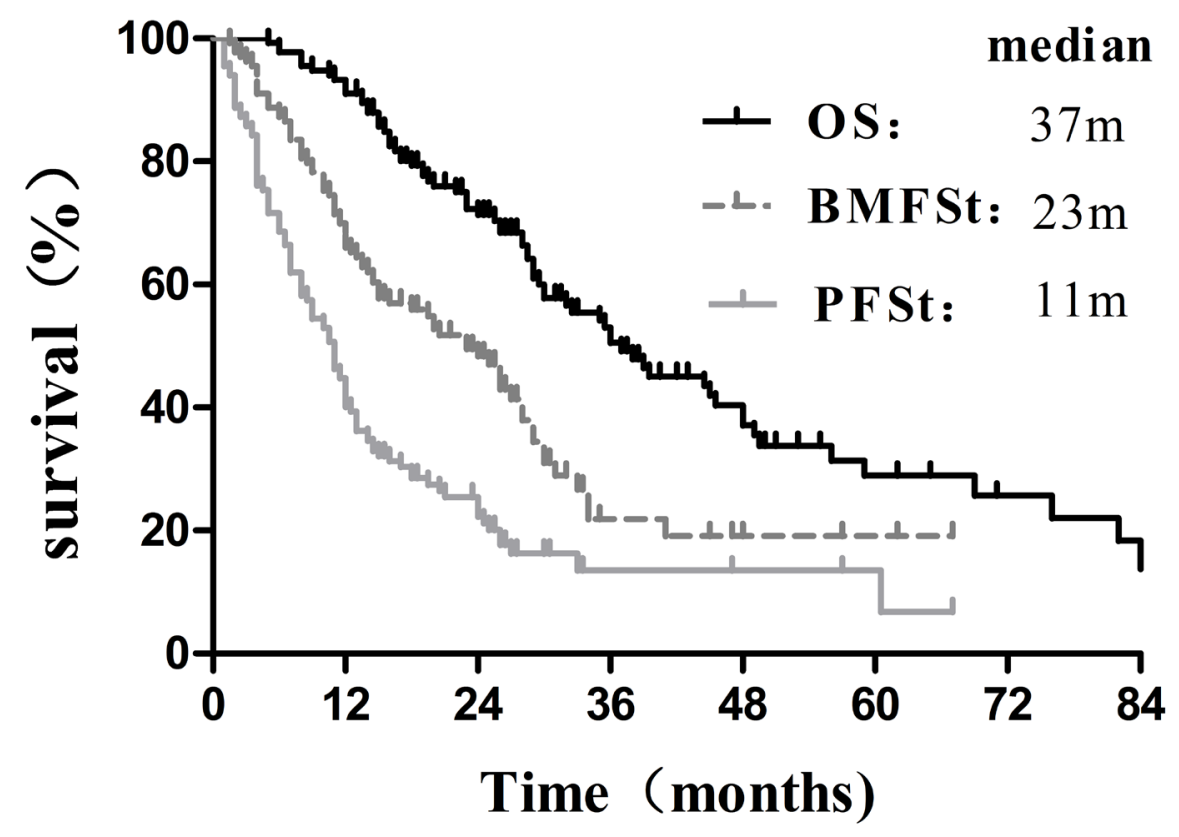

Figure 1: Overall survival, Brain-metastasis-free survival of TKIs and Progression-free survival of TKIs for 134 patients with EGFR-mutated advanced lung adenocarcinoma. 
Table 1: Clinical characteristics of patients with EGFR-TKIs therapy

\begin{tabular}{|c|c|c|}
\hline Characteristics & NO. & $\%$ \\
\hline \multicolumn{3}{|l|}{ Age (years old) } \\
\hline$>53$ & 85 & 63.4 \\
\hline$\leq 53$ & 49 & 36.6 \\
\hline \multicolumn{3}{|l|}{ Gender } \\
\hline Male & 59 & 44.0 \\
\hline Female & 75 & 56.0 \\
\hline \multicolumn{3}{|l|}{ KPS score } \\
\hline$\geq 80$ & 117 & 87.3 \\
\hline$<80$ & 17 & 12.7 \\
\hline \multicolumn{3}{|l|}{ Smoking status } \\
\hline Yes & 31 & 23.1 \\
\hline No & 103 & 76.9 \\
\hline \multicolumn{3}{|l|}{ CEA (ng/mL) } \\
\hline$>23$ & 77 & 57.5 \\
\hline$\geq 23$ & 57 & 42.5 \\
\hline \multicolumn{3}{|l|}{ EGFR-TKIs treatment } \\
\hline First-line & 27 & 20.2 \\
\hline Second-line & 72 & 53.7 \\
\hline Third-line or multi-line & 35 & 26.1 \\
\hline \multicolumn{3}{|c|}{ No. of extracranial metastasis } \\
\hline 0 & 19 & 14.2 \\
\hline 1 & 74 & 55.2 \\
\hline 2 or more & 41 & 30.6 \\
\hline \multicolumn{3}{|l|}{ Type of EGFR mutations } \\
\hline Exon 19 deletion mutations & 78 & 58.2 \\
\hline Exon 21 point mutations & 56 & 41.8 \\
\hline \multicolumn{3}{|l|}{ Type of EGFR-TKIs } \\
\hline Erlotinib & 79 & 59.0 \\
\hline Gefitinib & 55 & 41.0 \\
\hline \multicolumn{3}{|l|}{ Brain metastasis } \\
\hline Yes & 34 & 25.4 \\
\hline No & 100 & 74.6 \\
\hline
\end{tabular}

Abbreviations: KPS, karnofsky performance status; CEA, carcinoembryonic antigen; EGFR-TKIs, Epidermal growth factor receptor tyrosine kinase inhibitors

38.0months (range: 4.0-91.3 months). At the end of follow-up, 41 patients $(30.6 \%)$ were still alive, whereas 93 patients $(69.4 \%)$ had died. For these patients, median OS, median BMFS and median $\mathrm{PFS}_{\mathrm{t}}$ were 37.0 months (95\% CI: 27.1-46.9 months), 23.0 months (95\% CI: 17.428.6 months) and 11.0 months (95\% CI: 9.4-12.6 months), respectively (Figure 1). Additionally, the survival in these patients who didn't develop BM was significantly superior to that in patients who developed BM $(p=0.017$, Figure 2 ), according to the calculation from the date of starting EGFR-TKIs treatment.

\section{BM and post-treatment}

Thirty-four patients $(34 / 134,25.4 \%)$ developed BM during the course of EGFR-TKIs therapy. Among them, patients with symptomatic BM and asymptomatic BM were $28(82.4 \%)$ and $6(17.6 \%)$, respectively. In addition, 31 patients $(31 / 34,91.2 \%)$ receiving chemotherapy first developed BM during EGFR-TKIs therapy later. The median time for the occurrence of BM was 9 months (range: 1.5-25.0 months) on the basis of evaluation from the initiation of EGFR-TKIs treatment. The cumulative incidence of BM in all patients at 1 year and 2 years was $21.8 \%$ and $28.5 \%$, respectively. The actuarial risk for the occurrence of BM was $38.8 \%$ (19/49) in patients with age $\leq 53$ years and $17.6 \%(15 / 85)$ in patients with age $>53$ years; $33.9 \%(19 / 56)$ in patients harboring exon 21 point mutations and $19.2 \%(15 / 78)$ with exon 19 deletion mutations; and 38.6\% (22/57) in patients with serum CEA $\geq 23 \mathrm{ng} / \mathrm{mL}$ and $15.6 \%(12 / 77)$ in the group with serum $\mathrm{CEA}<23 \mathrm{ng} / \mathrm{mL}$.

For 34 patients who developed BM during the course 
Table 2: Univariate and multivariate analyses for the factors associated with actuarial risks for BM

\begin{tabular}{|c|c|c|c|c|c|c|c|}
\hline \multirow[t]{2}{*}{ Factors } & \multicolumn{4}{|c|}{$\begin{array}{l}\text { Univariate analysis } \\
\text { Incidence of BM (\%) }\end{array}$} & \multicolumn{3}{|c|}{$\begin{array}{l}\text { Multivariate analysis } \\
\text { Incidence of BM (\%) }\end{array}$} \\
\hline & $1 \mathrm{y}$ & $2 y$ & $\mathrm{X}^{2}$ & $P$ & HR & $95 \%$ CI & $P$ \\
\hline \multicolumn{8}{|l|}{ Age (years old) } \\
\hline$\leq 53$ & 32.9 & 40.9 & & & & & \\
\hline$>53$ & 15.2 & 21.3 & 7.236 & 0.007 & 2.751 & $1.326-5.707$ & 0.007 \\
\hline \multicolumn{8}{|l|}{ Gender } \\
\hline Male & 27.9 & 34.3 & & & & & \\
\hline Female & 17.1 & 24.2 & 1.339 & 0.247 & & & \\
\hline \multicolumn{8}{|l|}{ KPS score } \\
\hline$\geq 80$ & 19.2 & 25.7 & & & & & \\
\hline$<80$ & 39.6 & 47.1 & 2.957 & 0.086 & & & \\
\hline \multicolumn{8}{|l|}{ Smoking status } \\
\hline Yes & 37.6 & 48.1 & & & & & \\
\hline No & 17.5 & 23.0 & 5.334 & 0.021 & 1.918 & $0.886-4.149$ & 0.098 \\
\hline \multicolumn{8}{|l|}{ CEA (ng/mL) } \\
\hline$<23$ & 13.0 & 18.0 & & & & & \\
\hline$\geq 23$ & 33.9 & 44.5 & 12.15 & 0.001 & 3.197 & $1.512-6.758$ & 0.002 \\
\hline \multicolumn{8}{|l|}{ EGFR-TKIs treatment } \\
\hline First-line & 12.2 & 12.2 & & & & & \\
\hline Second-line & 21.1 & 24.5 & & & & & \\
\hline Third-line or multi-line & 31.2 & 49.8 & 7.343 & 0.025 & 1.276 & $0.722-2.257$ & 0.401 \\
\hline \multicolumn{8}{|l|}{ No. of extracranial metastasis } \\
\hline 0 & 10.5 & 18.0 & & & & & \\
\hline 1 & 22.8 & 26.0 & & & & & \\
\hline 2 or more & 25.8 & 41.3 & 1.758 & 0.415 & & & \\
\hline \multicolumn{8}{|l|}{ Type of EGFR mutations } \\
\hline Exon 19 deletion mutations & 17.8 & 20.1 & & & & & \\
\hline Exon 21 point mutations & 28.1 & 41.3 & 5.055 & 0.025 & 2.769 & $1.355-5.659$ & 0.005 \\
\hline
\end{tabular}

Abbreviations: BM, brain metastasis

of EGFR-TKIs therapy, 26 patients (76.5\%) experienced BM only, and 8 patients (23.5\%) experienced BM and extracranial disease progression. Among them, 18 patients $(52.9 \%)$ received radiation therapy (RT) plus continuous EGFR-TKIs, 8 patients (23.5\%) switched to chemotherapy, 6 patients $(17.6 \%)$ received continuous EGFR-TKIs and deferring RT until intracranial progression, and 2 patients $(5.9 \%)$ received continuous EGFR-TKIs plus supportive care. With regard to 100 patients who didn't develop BM during the course of EGFR-TKIs therapy, excluding 21 cases with stable disease, the most common sites of extracranial disease progression were thoracic cavity (60.8\%), bone (22.8\%), liver (8.9\%) and others (13.9\%). Of them, 42 patients $(53.2 \%)$ switched to chemotherapy, 27 patients (34.2\%) received local therapy plus continuous EGFR-TKIs, and 10 patients (12.7\%) received continuous EGFR-TKIs. As a result, the median duration of EGFRTKIs therapy was 13.5 months (range: 1.0-67.0 months). Finally, the treatment responses were evaluated according to the RECIST 1.1 guidelines and categorized as complete response (CR), partial response (PR), stable disease (SD), and progressive disease (PD).

\section{Risk factors for BM development}

Several clinical factors were observed to be associated with actuarial risk of developing BM by univariate and multivariate analyses in Table 2. In univariate analysis, the age of patients, type of EGFR mutations, serum CEA level, smoking status and treatment timing of EGFR-TKIs were associated with an increased risk of developing BM. The cumulative incidence of BM at 1 year and 2 years in patients with age $\leq 53$ years was $32.9 \%$ and $40.9 \%$, respectively, which was significantly higher compared with $15.2 \%$ and $21.3 \%$ respectively in patients with age $>53$ years ( $p=0.007$; Figure 3 ). The 1 - year and 2 -year actuarial risk of developing BM in patients with exon 21 point mutations was $28.1 \%$ and $41.3 \%$, respectively, which was considerably higher compared with $17.8 \%$ and $20.1 \%$ respectively in patients harboring exon 19 deletion mutations ( $p=0.025$; Figure 4). The cumulative incidence of BM at 1 year and 2 years in patients with serum CEA $\geq 23 \mathrm{ng} / \mathrm{mL}$ was $33.9 \%$ and $44.5 \%$, respectively, which was dramatically higher compared with $13.0 \%$ and $18.0 \%$ respectively in patients 


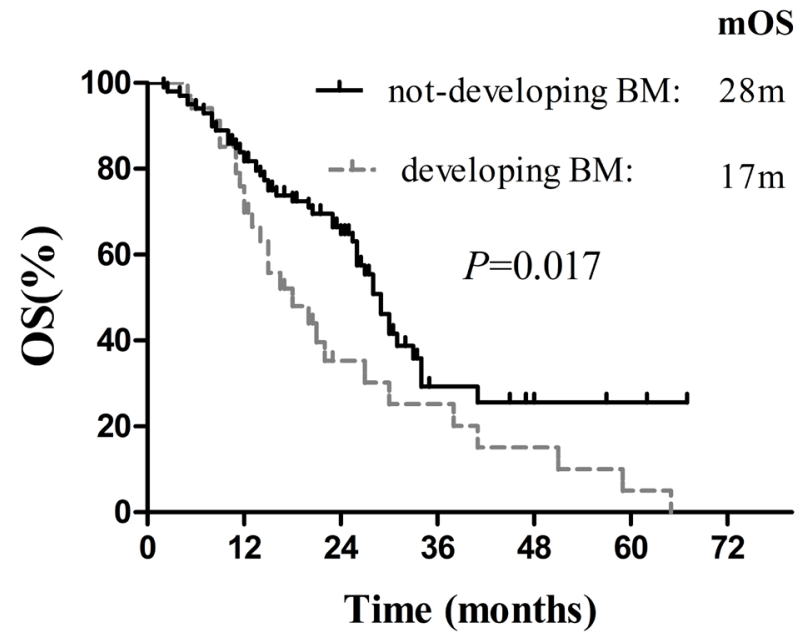

Figure 2: Comparison of the survival between patients with BM development and without BM development.

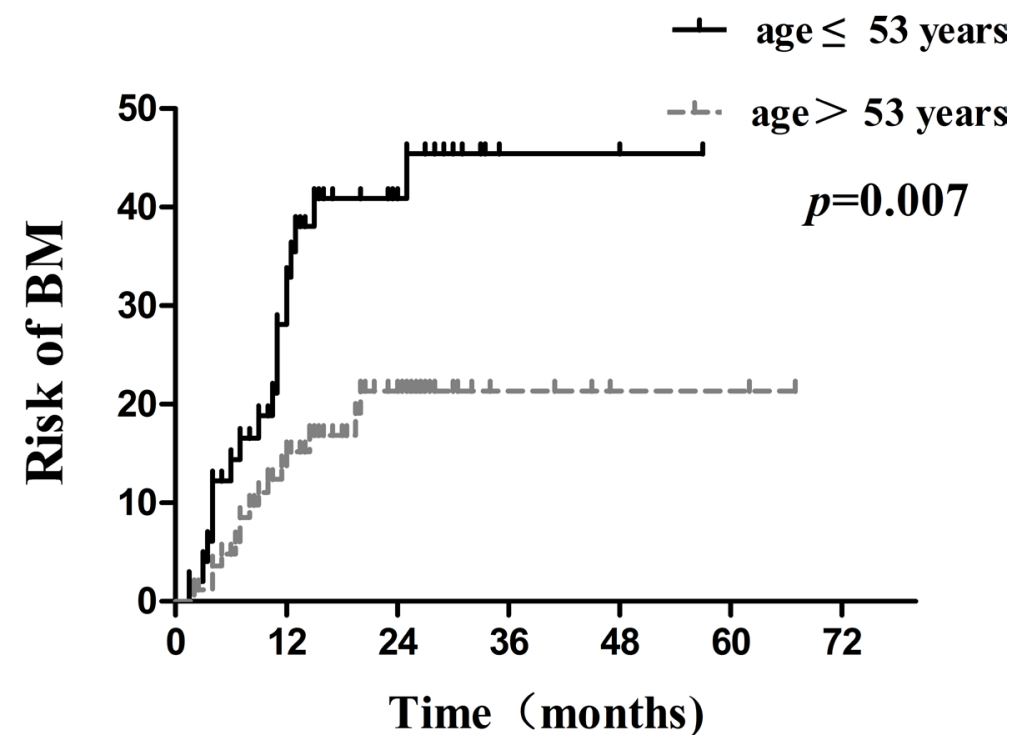

Figure 3: Comparison of the actuarial risk for developing BM between age $>53$ years and age $\leq 53$ years.

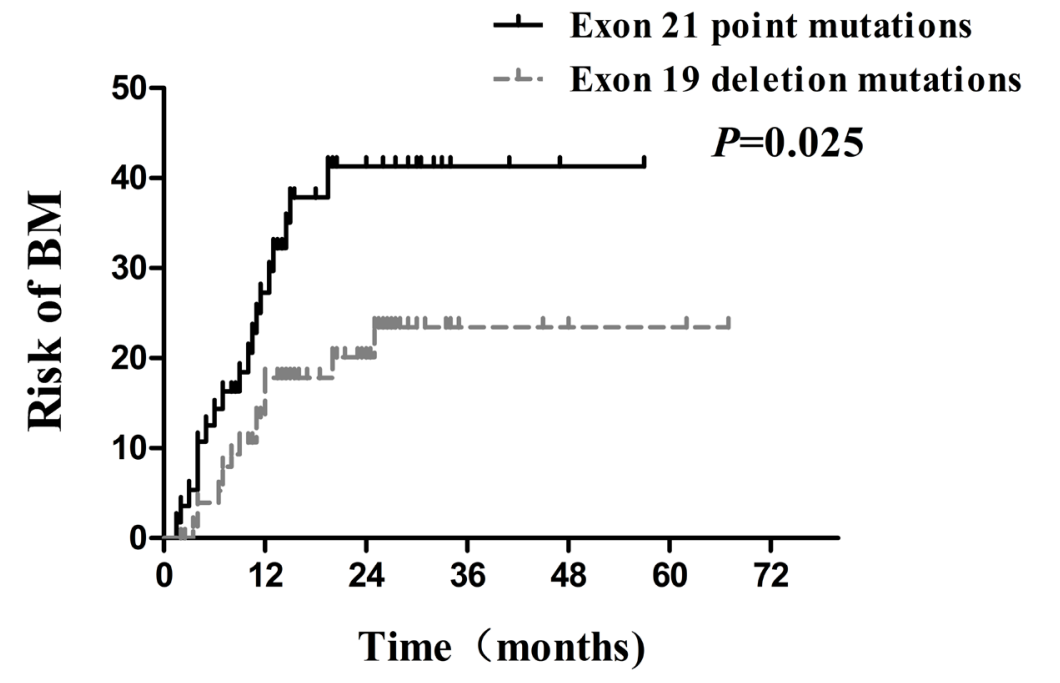

Figure 4: Comparison of the actuarial risk for developing BM between exon 21 point mutations and exon 19 deletion mutations. 
with serum CEA $<23 \mathrm{ng} / \mathrm{mL}(p=0.001$; Figure 5 ). And the 1-year and 2 -year actuarial risk of developing $\mathrm{BM}$ in patients with smoking was $37.6 \%$ and $48.1 \%$ , respectively, which is higher compared with $17.5 \%$ and $23.0 \%$ respectively in patients with non-smoking ( $p=0.021$; Figure 6A). Moreover, the actuarial risk of developing BM at 1 year and 2 years was $12.2 \%$ and $12.2 \%$, respectively, in patients with first-line treatment; $21.1 \%$ and $24.5 \%$, respectively, in patients with secondline treatment; and $31.2 \%$ and $49.8 \%$, respectively, in patients with third-line or multi-line treatment ( $p=0.025$, Figure 6B).

Multivariate analysis indicated that age $\leq 53$ years (HR: 2.751, $95 \%$ CI: 1.326-5.707; $p=0.007$ ), exon 21 point mutations (HR: 2.769, $95 \% \mathrm{CI}: 1.355-5.659 ; p=$ 0.005 ), and serum CEA $\geq 23 \mathrm{ng} / \mathrm{mL}$ (HR: 3.197, $95 \%$ CI: $1.512-6.758 ; p=0.002$ ) were independent high-risk factors of developing BM. In addition, further analysis and correlation on the independent risk factors above were conducted. For patients with no $(n=30), 1(n=52)$ and 2 or 3( $n=52)$ risk factors, the actuarial risk of developing $\mathrm{BM}$ at 1 year and 2 years were $6.9 \%$ and $6.9 \%, 17.7 \%$ and $21.0 \%, 34.7 \%$ and $49.4 \%$, respectively $(p=0.000$, Figure 7 ). Obviously, patients with more risk factors are at a higher risk for developing BM.

\section{DISCUSSION}

During the past decade, the advancement of EGFRTKIs revolutionarily transformed the landscape of treatment and prognosis in advanced lung adenocarcinoma, but BM remains a therapeutically challenging issue [24]. $\mathrm{BM}$ from advanced lung adenocarcinoma, is a common reason leading to the failure of treatment, which often predicts poor prognosis $[3,4,25]$. On the other hand, there are still some patients developing BM during the course of EGFR-TKIs therapy, although EGFR-TKIs are effective for EGFR-mutated NSCLC patients with BM [12-14]. As reported in our study, thirty-four patients (34/134, 25.4\%) developed BM during the course of EGFR-TKIs therapy. Moreover, patients had the tendency to live longer with more time to develop BM due to EGFR-TKIs therapy [26]. Therefore, it is urgent to identify the patients at the highest risk of BM for PCI, which may bring improvement in quality of life and survival for these patients.

Several studies have demonstrated that risk factors for BM in NSCLC [18-22], but there were no reports on the risk factors for developing BM during the course of EGFR-TKIs therapy from EGFR-mutated advanced lung adenocarcinoma. In the present study, multivariate analysis indicated that the age $\leq 53$ years was correlated with an increased risk of developing BM. Indeed, younger age was regarded as a high risk factor for BM in several studies. Bajard et al. [27] reported that age $\leq 62$ years was a risk factor for BM (HR: 2.5, $95 \%$ CI: 1.33-4.76; $p=0.004$ ). Ceresoli et al. [28] assumed that age $<60$ years was associated with a higher incidence rate of BM (HR: 1.26, 95 \% CI: 1.03-1.53; $p=0.03$ ). Similarly, Dimitropoulos et al. [29] confirmed that younger age $(60.8 \pm 8.9$ years $)$ was associated with a higher BM possibility (HR: 0.91; 95\% CI:0.87-0.96; $p<0.001)$. In spite of the difference in the definition of younger age, to some extent, the results of these studies are consistent. So far, the reason that younger patients are at a higher risk for BM is still unclear. Younger patients with a higher risk for BM may be due to a more aggressive course of disease [30] and some biological factors, including high $\mathrm{Ki}-67$ level, increased expression of vascular endothelial growth factor (VEGF) and so on $[31,32]$. Further investigations are required to identify the specific reasons that younger patients are more likely to develop BM.

$\leftarrow$ CEA $\geq 23 \mathrm{ng} / \mathrm{mL}$

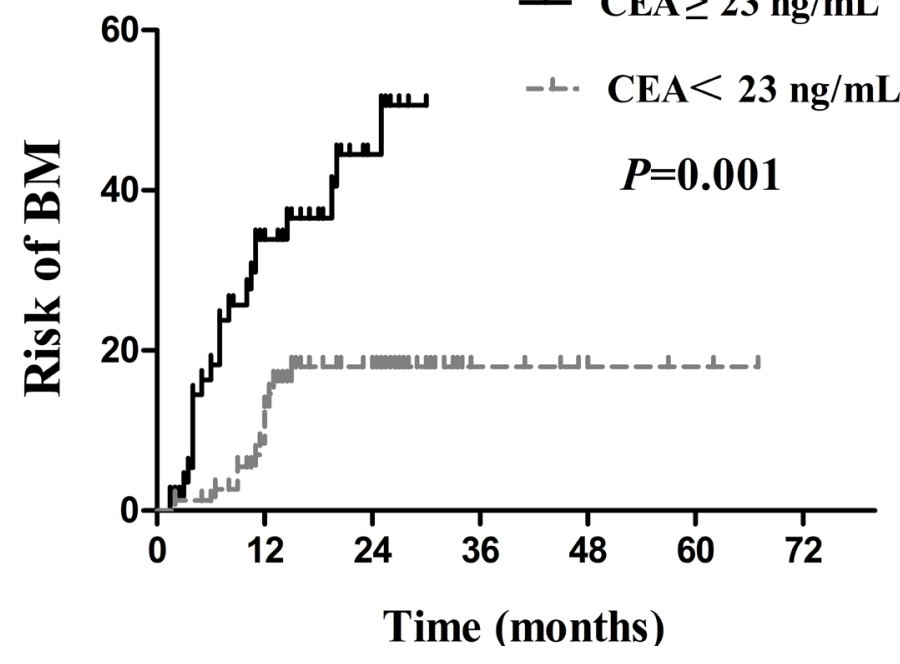

Figure 5: Comparison of the actuarial risk for developing BM between serum CEA $\geq 23 \mathrm{ng} / \mathrm{mL}$ and serum $\mathrm{CEA}<23$ ng/mL. 

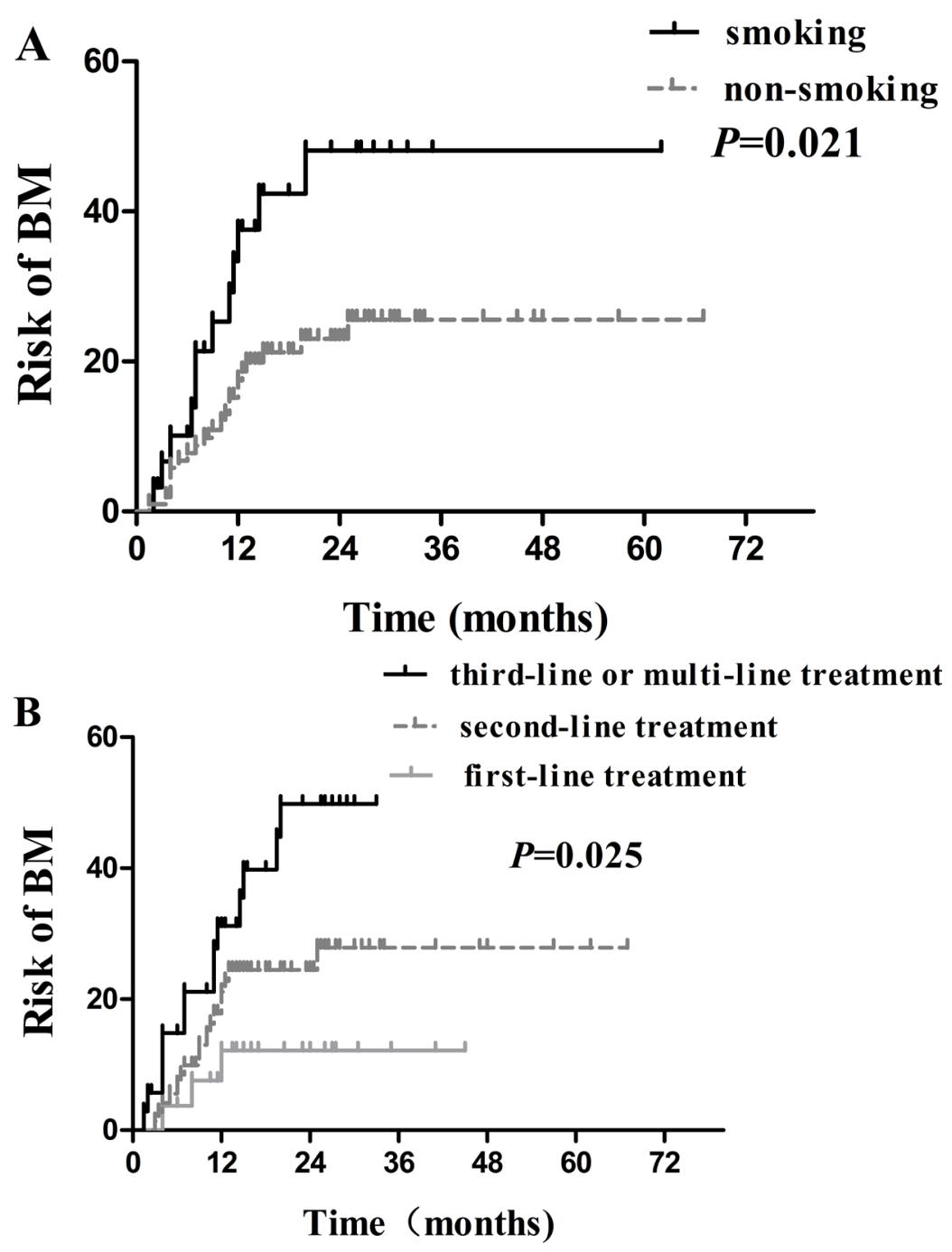

Figure 6: Comparison of the actuarial risk for developing BM. A. between smoking and non-smoking; B. among patients with EGFR-TKIs first-line treatment, second-line treatment, and third-line or multi-line treatment.

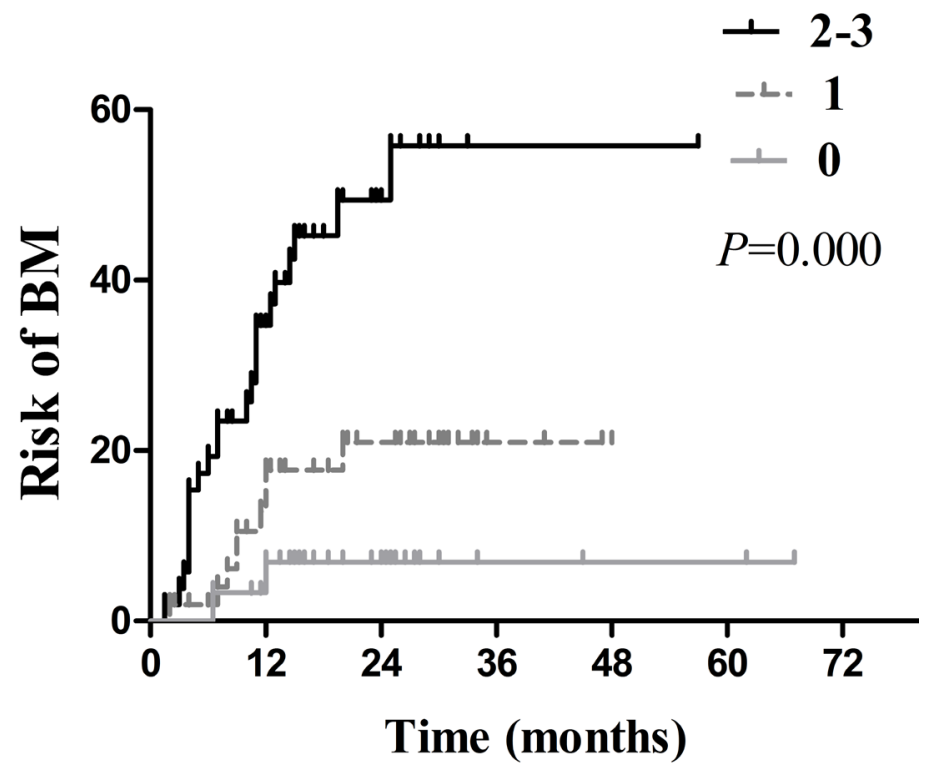

Figure 7: Comparison of the actuarial risk for developing BM among patients with different numbers of risk factors. 
Recently, with the increasing availability of EGFRTKIs, the type of EGFR mutations has become the research hotspot. Exon 19 deletion mutations and exon 21 point mutations, the most common EGFR mutations, account for approximately $85 \%$ of all mutations of EGFR [33]. At present, several previous studies have shown that EGFR exon 19 or 21 mutations are different predictive markers for the response to EGFR-TKIs. It is well known that two open-label, randomized, phase III trials (LUXLung 3 and LUX-Lung 6) showed first-line afatinib improved OS compared with chemotherapy, especially in patients with exon 19 deletion mutation from lung adenocarcinoma but not in patients with exon 21 point mutations [10]. Sekine et al. [34] reported that the patients with exon 19 deletion mutations presented longer OS than those with exon 21 point mutations among NSCLC patients with erlotinib treatment after BM diagnosis ( $p$ $=0.019$ ). Furthermore, EGFR exon 19 or 21 mutations are now well recognized as different prognostic markers for NSCLC. A recent retrospective study by Li et al. [35] showed that exon 19 deletion, an independent prognostic factor, was associated with significantly longer survival in NSCLC with BM ( HR: 0.558, 95 \% CI: 0.325-0.957; $p=0.034$ ). According to our knowledge, previous studies usually focused on the predictive value of EGFR exon19 or 21 mutations in terms of the response to EGFR-TKIs and prognosis in NSCLC. In contrast, we investigated risk factors of $\mathrm{BM}$ development based on the type of EGFR mutations. In the present study, multivariate analysis demonstrated that exon 21 point mutations was an independent high-risk factor for BM (HR: 2.769, 95 $\%$ CI: $1.355-5.659 ; p=0.005)$, which in turn supported previous studies. However, Heon et al. [36] retrospectively explored the risk of CNS progression in patients with IIIB/IV NSCLC initially treated with EGFR-TKIs, and drew an opposite conclusion that a higher risk of CNS progression was observed in patients bearing exon 19 deletion mutations. Accordingly, authoritative prospective investigations are required to validate the findings. The reasons for the significant difference between EGFR exon 19 deletion and 21 point mutations are still uncertain. One of the possible reasons for this finding may be attributed to different clinical characteristics and pathogenesis between exon 19 deletion and 21 point mutations, suggesting they were two kinds of NSCLCs [37]. Alternatively, exon 19 deletion mutations may lead to a better control of subclinical lesions of BM, which may be the explanation of lower incidence of BM.

CEA played an important role in the diagnosis, follow-up and prognosis of lung adenocarcinoma as an important tumor marker [38]. High serum CEA level was connected with tumor recurrence and metastases in resected NSCLC [39]. As the relationship between serum CEA levels and BM, Arrieta et al. [21] demonstrated that serum CEA $\geq 40 \mathrm{ng} / \mathrm{mL}$ is a risk factor for developing BM (HR:1.5, 95\%CI:1.09-2.2; $p=0.014$ ), particularly in lung adenocarcinoma. Consistent with the result above, our analysis for 134 patients with EGFR-mutated advanced lung adenocarcinoma also demonstrated that $\mathrm{CEA} \geq 23 \mathrm{ng} / \mathrm{mL}$ was associated with an increased risk for developing BM. Taken together, high serum CEA level was not only related to tumor charge, but also suggested as a more invasive phenotype $[21,38]$. Of note, the level of CEA in our study was measured prior to the initiation of EGFR-TKIs treatment, thus it can more accurately reflect the occurrence of BM during the course of EGFR-TKIs therapy.

There were no sufficient studies available regarding to treatment timing of EGFR-TKIs, especially in advanced NSCLC with BM. Koo et al. [39] reported that EGFR-TKIs are effective for EGFR-mutated lung adenocarcinoma, regardless of treatment timing. Among these 134 patients receiving EGFR-TKIs therapy in our study, 27 (20.2\%), 72 (53.7\%), and 35 (26.1\%) patients received EGFR-TKIs as first-line, second-line, and thirdline or multi-line treatment, respectively. The univariate analysis indicated that patients with third-line or multi-line treatment were more likely to develop BM $(p=0.025)$, whereas multivariate analysis failed to show a statistical difference in the association between treatment timing of EGFR-TKIs and BM development $(p=0.401)$. Whether there were some differences among first-line, secondline, and third-line or multi-line treatment in terms of BM development needs further exploration. Similar to treatment timing of EGFR-TKIs, only univariate analysis showed that smoking status was associated with a higher risk of developing BM. A study from Jain et al. [40] revealed that smoking status had negative impact on survival in a cohort of 211 patients with EGFR-mutated advanced lung adenocarcinoma treated with first line EGFR TKIs. Overall, published data are too limited to draw a definitive conclusion.

Currently, the profound significance of PCI in patients with limited SCLC and extensive SCLC has been established [41]. However, the survival advantage conferred by PCI in NSCLC remains controversial [17]. In this study, we struggled to identify the potential patients at the highest risk for BM during the course of EGFR-TKIs therapy for PCI, and the rationality of the investigation could be explained as follows. First, EGFR-TKIs as a systemic treatment have led to significant improvements in the control of locoregional and extracranial disease. Second, the combination of EGFR-TKIs and radiotherapy could result in favorable synergistic effects, including the radiosensitizing effect of TKIs and the opening of BBB by radiation [42]. Furthermore, the total dosage in the range of 20 to $40 \mathrm{~Gy}$ may lead to the maximal opening of BBB, with the tolerable side effects [43]. Given all these considerations, PCI might bring survival benefit for targeted population.

In addition, median survival was 27 months (95\% CI: 24.4-29.6 months) based on the calculation from the 
initiation of EGFR-TKIs treatment. The patients without BM development during the course of EGFR-TKIs therapy showed clear OS superiority over those with BM development. ( $p=0.017$,Figure 2). Not surprisingly, for patients with more risk factors for BM, there was an obvious declining trend in OS $(p=0.026)$. However, similar to other retrospective analyses, our study has several limitations. For example, EGFR mutation status was assessed using tissue specimens from primary and metastatic lesions, rather than BM. Importantly, the discordance rate of EGFR mutation status between primary tumors and corresponding metastases was reported to be $27-28 \%$ [44], which may influence our findings to some extent. What's more, selection bias and heterogeneity in the enrollment may lead to a relatively longer OS, which was measured from the date of diagnosis. Undoubtedly, longer OS was mainly caused by 32 patients with postoperative recurrence or metastases.

\section{MATERIALS AND METHODS}

\section{Patients}

A total of 238 patients with EGFR-mutated advanced lung adenocarcinoma were treated with EGFRTKIs at the Shandong Cancer Hospital and Institute between January 2008 and December 2012. The exclusion criteria were as follows: 1) BM identified before EGFRTKIs therapy (90 patients); 2) EGFR-TKIs treatment less than one month (6 patients), and 3) incomplete clinical data to follow up (8 patients). Thus, 134 eligible patients were enrolled in the retrospective study, including 102 patients with stage IIIB-IV at initial diagnosis and 32 patients with postoperative recurrence or metastases. These patients underwent a comprehensive assessment before EGFR-TKIs treatment, including physical examination, laboratory analysis, pathological type, EGFR mutation testing, and TNM stage. All patients had negative results of enhanced magnetic resonance imaging (MRI) or computed tomography (CT) scan of brain before EGFR-TKIs therapy; among these, 119 patients (88.8\%) received brain enhanced MRI scan and 15 patients $(11.2 \%)$ received brain enhanced CT scan. Generally, these patients periodically underwent a reexamination every two months, which composed of thoracic CT scan, abdomen B-ultrasound examination, brain enhanced MRI scan, and other necessary examinations based on their conditions. Of all the patients, 34 cases were identified to have BM during the course of EGFR-TKIs treatment. Additionally, EGFR mutations were detected by real-time fluorescent quantitative PCR (ARMS), using tissue specimens from primary and metastatic lesions. The study protocol was approved by the Ethics Committee of the Shandong Cancer Hospital and Institute.

\section{Treatment}

Totally 134 patients with EGFR-mutated advanced lung adenocarcinoma received different treatment regimens before EGFR-TKIs therapy. Among these patients, 32 cases underwent postoperative recurrence or metastases and others were advanced NSCLC at the first present. There were 27 patients receiving EGFRTKIs treatment as their first-line therapy, whereas the remaining preferred chemotherapy initially, with or without radiotherapy. For EGFR-TKIs therapy, gefitinib and erlotinib were continuously administered at the doses of 250 and $150 \mathrm{mg} /$ day, respectively, until progression of disease (PD) or intolerable side effects.

\section{Statistical analysis}

Brain-metastasis-free survival of TKIs $\left(\right.$ BMFS $\left._{t}\right)$ was defined as the time from the date of starting EGFRTKIs treatment to the date of BM or to the date of last follow-up without the occurrence of BM. Progression-free survival of TKIs (PFS $)_{t}$ was defined as the time from the initiation of EGFR-TKIs therapy to disease progression or death. Overall survival (OS) was measured from the date of diagnosis to the date of death from any causes or the last known date that the patient was alive. The number of extracranial metastases was calculated by the region, such as thoracic cavity, bone, liver, adrenal glands and so on. In addition, the cut-off point of age and CEA were determined by ROC curve. The cumulative incidence of BM and survival were calculated by the Kaplan-Meier method. All statistically significant variables in univariate analysis were entered into the multivariate Cox regression analysis. The Log-rank test was used to compare the difference between groups, and two-sided $p$ values $<0.05$ was considered statistically significant.

\section{CONFLICTS OF INTERESTS}

The authors declare no any conflicts of interest in this work.

\section{GRANT SUPPORT}

This study was supported by the Natural Science Foundation of Shandong Province to Hui Zhu (Grant No. ZR2015HM040) and Special Fund for National Health and Family Planning Commission: specification and application of image-guided individualized radiotherapy in NSCLC (Grant No. 201402011).

\section{REFERENCES}

1. Torre LA, Bray F, Siegel RL, Ferlay J, Lortet-Tieulent J and Jemal A. Global cancer statistics, 2012. CA. 2015; 
65(2):87-108. doi: 10.3322/caac.21262.

2. Saad AG, Yeap BY, Thunnissen FB, Pinkus GS, Pinkus JL, Loda M, Sugarbaker DJ, Johnson BE and Chirieac LR. Immunohistochemical markers associated with brain metastases in patients with nonsmall cell lung carcinoma. Cancer. 2008; 113(8):2129-2138. doi: 10.1002/cncr.23826.

3. Arrieta O, Villarreal-Garza C, Zamora J, Blake-Cerda M, de la Mata MD, Zavala DG, Muniz-Hernandez S and de la Garza J. Long-term survival in patients with non-small cell lung cancer and synchronous brain metastasis treated with whole-brain radiotherapy and thoracic chemoradiation. Radiation oncology. 2011; 6:166. doi: 10.1186/1748-717X6-166.

4. Gaspar LE, Mehta MP, Patchell RA, Burri SH, Robinson PD, Morris RE, Ammirati M, Andrews DW, Asher AL, Cobbs CS, Kondziolka D, Linskey ME, Loeffler JS, et al. The role of whole brain radiation therapy in the management of newly diagnosed brain metastases: a systematic review and evidence-based clinical practice guideline. Journal of neuro-oncology. 2010; 96(1):17-32. doi: 10.1007/s11060009-0060-9.

5. Fukuoka M, Wu YL, Thongprasert S, Sunpaweravong P, Leong SS, Sriuranpong V, Chao TY, Nakagawa K, Chu DT, Saijo N, Duffield EL, Rukazenkov Y, Speake G, et al. Biomarker analyses and final overall survival results from a phase III, randomized, open-label, first-line study of gefitinib versus carboplatin/paclitaxel in clinically selected patients with advanced non-small-cell lung cancer in Asia (IPASS). Journal of clinical oncology. 2011; 29(21):28662874. doi: 10.1200/JCO.2010.33.4235.

6. Wu YL, Chu DT, Han B, Liu X, Zhang L, Zhou C, Liao M, Mok T, Jiang H, Duffield E and Fukuoka M. Phase III, randomized, open-label, first-line study in Asia of gefitinib versus carboplatin/paclitaxel in clinically selected patients with advanced non-small-cell lung cancer: evaluation of patients recruited from mainland China. Asia-Pacific journal of clinical oncology. 2012; 8(3):232-243. doi: 10.1111/j.1743-7563.2012.01518.x.

7. Zhou C, Wu YL, Chen G, Feng J, Liu XQ, Wang C, Zhang S, Wang J, Zhou S, Ren S, Lu S, Zhang L, Hu C, Hu C, Luo Y, Chen L, et al. Erlotinib versus chemotherapy as first-line treatment for patients with advanced EGFR mutation-positive non-small-cell lung cancer (OPTIMAL, CTONG-0802): a multicentre, open-label, randomised, phase 3 study. The Lancet Oncology. 2011; 12(8):735-742. doi: 10.1016/S1470-2045(11)70184-X.

8. Inoue A, Kobayashi K, Maemondo M, Sugawara S, Oizumi S, Isobe H, Gemma A, Harada M, Yoshizawa H, Kinoshita I, Fujita Y, Okinaga S, Hirano H, et al. Updated overall survival results from a randomized phase III trial comparing gefitinib with carboplatin-paclitaxel for chemo-naive nonsmall cell lung cancer with sensitive EGFR gene mutations (NEJ002). Annals of oncology. 2013; 24(1):54-59. doi: 10.1093/annonc/mds214.

9. Sequist LV, Yang JC, Yamamoto N, O’Byrne K, Hirsh
V, Mok T, Geater SL, Orlov S, Tsai CM, Boyer M, Su WC, Bennouna J, Kato T, et al. Phase III study of afatinib or cisplatin plus pemetrexed in patients with metastatic lung adenocarcinoma with EGFR mutations. Journal of clinical oncology. 2013; 31(27):3327-3334. doi: 10.1200/ JCO.2012.44.2806.

10. Yang JC, Wu YL, Schuler M, Sebastian M, Popat S, Yamamoto N, Zhou C, Hu CP, O'Byrne K, Feng J, Lu $\mathrm{S}$, Huang Y, Geater SL, et al. Afatinib versus cisplatinbased chemotherapy for EGFR mutation-positive lung adenocarcinoma (LUX-Lung 3 and LUX-Lung 6): analysis of overall survival data from two randomised, phase 3 trials. The Lancet Oncology. 2015; 16(2):141-151.

11. Sperduto PW, Yang TJ, Beal K, Pan HY, Brown PD, Ho YY, Yeh N, Gaspar LE, Braunstein SE and Sneed PK. The effect of gene mutations on survival in patients with adenocarcinoma of the lung following the development of brain metastases. International Journal of Radiation Oncology Biology Physics. 2015; 93(3):S37-S38.

12. Fan $\mathrm{Y}, \mathrm{Xu} \mathrm{X}$ and $\mathrm{Xie} \mathrm{C}$. EGFR-TKI therapy for patients with brain metastases from non-small-cell lung cancer: a pooled analysis of published data. OncoTargets and therapy. 2014; 7:2075-2084. doi: 10.2147/OTT.S67586.

13. Wu YL, Zhou C, Cheng Y, Lu S, Chen GY, Huang C, Huang YS, Yan HH, Ren S and Liu Y. Erlotinib as secondline treatment in patients with advanced non-small-cell lung cancer and asymptomatic brain metastases: a phase II study (CTONG-0803). Annals of Oncology. 2013; 24(4):993-999. doi: 10.1093/annonc/mds529.

14. Lynch TJ, Bell DW, Raffaella S, Sarada G, Okimoto RA, Brannigan BW, Harris PL, Haserlat SM, Supko JG and Haluska FG. Activating mutations in the epidermal growth factor receptor underlying responsiveness of non-small-cell lung cancer to gefitinib. New England Journal of Medicine. 2004; 350(21):2129-2139.

15. Britta W, Michael W, Ashfaque M, Sorensen BS, Susanne K, Leif S, Ebba N and Peter M. Erlotinib accumulation in brain metastases from non-small cell lung cancer: visualization by positron emission tomography in a patient harboring a mutation in the epidermal growth factor receptor. Journal of Thoracic Oncology. 2011; 6(7):12871289. doi: 10.1097/JTO.0b013e318219ab87.

16. Lee YJ, Choi HJ, Kim SK, Chang J, Moon JW, Park IK, $\mathrm{Kim} \mathrm{JH}$, Cho BC. Frequent central nervous system failure after clinical benefit with epidermal growth factor receptor tyrosine kinase inhibitors in Korean patients with nonsmallcell lung cancer. Cancer. 2010; 116(5):1336-1343. doi: 10.1002/cncr.24877.

17. Gore EM, Kyounghwa B, Wong SJ, Alexander S, Bonner JA, Schild SE, Gaspar LE, Bogart JA, Maria WW and Hak C. Phase III comparison of prophylactic cranial irradiation versus observation in patients with locally advanced non-small-cell lung cancer: primary analysis of radiation therapy oncology group study RTOG 0214. Journal of Clinical Oncology. 2011; 29(3):272-278. doi: 10.1200/ 
JCO.2010.29.1609.

18. Zhe J, Nan B, Jingbo W, Zhouguang H, Zefen X, Qinfu F, Zongmei Z, Dongfu C, Jima L and Jun L. Risk factors for brain metastases in locally advanced non-small cell lung cancer with definitive chest radiation. International Journal of Radiation Oncology Biology Physics. 2014; 89(2):330337. doi: 10.1016/j.ijrobp.2014.02.025.

19. Giovanni LC, Michele R, Giuseppe C, Angelo C, Stefano S, Paolo P, Angelo B, Piero Z and Eugenio V. Brain metastases in locally advanced non-small cell lung carcinoma after multimodality treatment: risk factors analysis. Cancer. 2002; 95(3):605-612.

20. Gaspar LE, Kari C, Albain KS, Eric V, Valerie R, Crowley JJ, Livingston RB and Gandara DR. Time from treatment to subsequent diagnosis of brain metastases in stage III non-small-cell lung cancer: a retrospective review by the Southwest Oncology Group. Journal of Clinical Oncology. 2005; 23(13):2955-2961.

21. Arrieta O, Saavedra-Perez D, Kuri R, Aviles-Salas A, Martinez L, Mendoza-Posada D, Castillo P, Astorga A, Guzman E and Garza JDL. Brain metastasis development and poor survival associated with carcinoembryonic antigen (CEA) level in advanced non-small cell lung cancer: a prospective analysis. Bmc Cancer. 2009; 9(16): 1-9.

22. Robnett TJ, Machtay M, ., Stevenson JP, Algazy KM and Hahn SM. Factors affecting the risk of brain metastases after definitive chemoradiation for locally advanced nonsmall-cell lung carcinoma. Journal of Clinical Oncology. 2001; 19(5):1344-1349.

23. Dong-Yeop S, Im Il N, Cheol Hyeon K, Sunhoo P, Heejong $\mathrm{B}$ and Sung Hyun Y. EGFR mutation and brain metastasis in pulmonary adenocarcinomas. Journal of Thoracic Oncology. 2014; 9(2):195-199.

24. Tang N, Guo J, Zhang QQ, Wang YL and Wang ZH. Greater efficacy of chemotherapy plus bevacizumab compared to chemo- and targeted therapy alone on non-small cell lung cancer patients with brain metastasis. Oncotarget. 2015; 7:3635-44. doi: 10.18632/oncotarget.6184.

25. Zhao X, Zhu G, Chen H, Yang P, Li F and Du N. Efficacy of icotinib versus traditional chemotherapy as first-line treatment for preventing brain metastasis from advanced lung adenocarcinoma in patients with epidermal growth factor receptor-sensitive mutation. Journal of Cancer Research \& Therapeutics. 2014; 10 :C155-159. doi: 10.4103/0973-1482.

26. Patel RR and Mehta MP. Targeted therapy for brain metastases: improving the therapeutic ratio. Clinical Cancer Research. 2007; 13(6):1675-1683.

27. Bajard A, Westeel V, Dubiez A, Jacoulet P, Pernet D, Dalphin JC and Depierre A. Multivariate analysis of factors predictive of brain metastases in localised non-small cell lung carcinoma. Lung Cancer. 2004; 46(3):357-360.

28. Giovanni LC, Michele R, Giuseppe C, Angelo C, Stefano S, Paolo P, Angelo B, Piero Z and Eugenio V.
Brain metastases in locally advanced nonsmall cell lung carcinoma after multimodality treatment: risk factors analysis. Cancer. 2002; 95(3):605-612.

29. Dimitropoulos C, Hillas G, Nikolakopoulou S, Kostara I, Sagris K, Vlastos F and Alchanatis M. Prophylactic cranial irradiation in non-small cell lung cancer patients: who might be the candidates? Cancer Management \& Research. 2011; 3(1):287-294.

30. D'Amico TA, Aloia TA, Moore MB, Conlon DH, Herndon JE, Kinch MS and Harpole DH. Predicting the sites of metastases from lung cancer using molecular biologic markers. Annals of Thoracic Surgery. 2001; 72(4):11441148.

31. Fidler IJ, Seiji Y, Ruo-Dan Z, Takahashi F and Bucana CD. The seed and soil hypothesis: vascularisation and brain metastases. Lancet Oncology. 2002; 3(1):53-57.

32. Saad AG, Yeap BY, Thunnissen FBJM, Pinkus GS, Pinkus JL, Massimo L, Sugarbaker DJ, Johnson BE and Chirieac LR. Immunohistochemical markers associated with brain metastases in patients with nonsmall cell lung carcinoma. Cancer. 2008; 113(8):2129-2138. doi: 10.1002/cncr.23826.

33. Fanny BV, Damien A, Michael C and Florence P. EGFR mutation status in brain metastases of non-small cell lung carcinoma. Journal of neuro-oncology. 2012; 111(1):1-10. doi: 10.1007/s11060-012-0990-5.

34. Sekine A, Satoh H, Iwasawa T, Tamura K, Hayashihara $\mathrm{K}$, Saito $\mathrm{T}$, Kato $\mathrm{T}$, Arai M, Okudela $\mathrm{K}$ and Ohashi $\mathrm{K}$. Prognostic factors for brain metastases from nonsmall cell lung cancer with EGFR mutation: influence of stable extracranial disease and erlotinib therapy. Medical Oncology. 2014; 31(10):1-10. doi: 10.1007/s12032-0140228-9.

35. Hongwei L, Xiaqin Z, Jianzhong C, Pengcheng S, Jianhong L, Xing S, Weihua Y, Songyan H, Yanfeng X and Yaohua W. Erratum to: Exon 19 deletion of epidermal growth factor receptor is associated with prolonged survival in brain metastases from non-small-cell lung cancer. Tumor Biology. 2015; 36(9):1-8. doi: 10.1007/s13277-015-3953-6.

36. Heon S, Yeap BY, Britt GJ, Costa DB, Rabin MS, Jackman DM, Johnson BE.Development of Central Nervous System Metastases in Patients with Advanced NonSmall Cell Lung Cancer and Somatic EGFR Mutations Treated with Gefitinib or Erlotinib. Clin Cancer Research. 2010;16(23):5873-5882.

37. Zhu JQ, Zhong WZ, Zhang GC, Li R, Zhang XC, Guo AL, Zhang YF, An SJ, Mok TS, Wu YL.Better survival with EGFR exon 19 than exon 21 mutations in gefi tinib-treated non-small cell lung cancer patients is due to diff erential inhibition of downstream signals. Cancer Lett. 2008; 265(2):307-17. doi: 10.1016/j.canlet.2008.02.064.

38. Icard P, Regnard JF, Essomba A, Panebianco V, Magdeleinat P, Levasseur P. Preoperative carcinoembryonic antigen level as a prognostic indicator in resected primary lung cancer. Annals of Thoracic Surgery. 1994; 58(3):811814. 
39. Koo DH, Kim KP, Choi CM, Lee DH, Lee JC, Lee JS, Jang SJ and Kim SW. EGFR-TKI is effective regardless of treatment timing in pulmonary adenocarcinoma with EGFR mutation.Cancer Chemotherapy \&Pharmacology.2014; 75(1):197-206. doi: 10.1007/s00280-014-2631-5.

40. Amit J, Cindy L, Eugene Mingjin G, David Zhihao N, Sing NQ, Kim AM, Angela T, Kian Sing C, Meng TW and Ravindran K. Impact of Smoking and Brain Metastasis on Outcomes of Advanced EGFR Mutation Lung Adenocarcinoma Patients Treated with First Line Epidermal Growth Factor Receptor Tyrosine Kinase Inhibitors. PloS one. 2015; 10(5): e0123587. doi: 10.1371/journal. pone. 0123587.

41. Auperin A, Arriagada R, Pignon JP, Le Pechoux C, Gregor A, Stephens RJ, Kristjansen PE, Johnson BE, Ueoka H, Wagner H, Aisner J: Prophylactic cranial irradiation for patients with small-cell lung cancer in complete remission: a meta-analysis of individual data from 987 patients. N Engl J Med 1999, 341:476-484.23-27.
42. Prakash C, Shyhmin H, Geetha V, Eric A, Sooryanarayana V, Tomlins SA, Chinnaiyan AM and Harari PM. Mechanisms of enhanced radiation response following epidermal growth factor receptor signaling inhibition by erlotinib (Tarceva). Cancer Research. 2005; 65(8):33283335 .

43. Qin D, Ou G, Mo H, Song Y, Kang G, Hu Y, Gu X. Improved efficacy of chemotherapy for glioblastoma by radiation-induced opening of blood-brain barrier: clinical results. International Journal of Radiation Oncology Biology Physics. 2001; 51(4):959-962.

44. Gow CH, Chang YL, Hsu YC, Tsai MF, Wu CT, Yu CJ, Yang CH, Lee YC, Yang PC and Shih JY. Comparison of epidermal growth factor receptor mutations between primary and corresponding metastatic tumors in tyrosine kinase inhibitor-naive non-small-cell lung cancer. Annals of Oncology. 2009; 20(4):696-702. doi: 10.1093/annonc/ mdn679. 